\title{
Muon Acceleration in a Superconducting Proton Linac ${ }^{*}$
}

\author{
Milorad Popovic, Fermi National Accelerator Laboratory, Batavia, IL USA and \\ Rolland P. Johnson, Muons, Inc., Batavia, IL USA
}

\begin{abstract}
1 Abstract
This note describes how a future Fermilab proton driver [1] based on TESLA superconducting linac modules can perform as both the source of protons to produce the muons and as the accelerator of the muons to be used for a neutrino factory or muon collider. Recent advances in muon cooling [2] have the promise of muon emittances that are compatible with the $1300 \mathrm{MHz}$ accelerating structures that are the basis for the ILC design. In the design described here, $\mathrm{H}^{-}$ions are accelerated to $8 \mathrm{GeV}$ in the superconducting Linac, then stripped, stored and bunched in a ring while the Linac cavities are rephased for muon accleleration. Then the protons are extracted from the ring to produce pions and muons which are cooled in a few hundred meters, accelerated to a few $\mathrm{GeV}$ and injected into the Linac at the $\beta=1$ point for acceleration to add $7 \mathrm{GeV}$. By recirculating the muons in the constant frequency section of such a proton driver Linac, even higher energies can be achieved quickly so that losses from muon decay are minimized. By adding additional refrigeration and RF power, the repetition rate of the Linac can be increased to make large increases in the average flux of a neutrino factory and the average luminosity of a muon collider.
\end{abstract}

\section{Introduction}

Fermilab has conducted design studies and cost estimates for both an $8 \mathrm{GeV}$ synchrotron and an $8 \mathrm{GeV}$ superconducting Linac as possible replacements for the Booster synchrotron, which now serves as the injector to the Fermilab Main Injector (FMI) and as the source of neutrinos for the MINIBOONE experiment. The recent decision to concentrate on cold technology for the International Linear Collider (ILC) has added an additional argument for the Booster replacement to be based on superconducting technology.

The large number of muons needed for a collider, or for a muon storage ring to produce neutrinos, requires a high-intensity proton driver for $\pi$-production, a highacceptance $\pi-\mu$ decay channel, a $\mu$-cooling system, a rapid acceleration system and a high-luminosity collider ring or muon storage ring with long straight decay or interaction region sections. Presently, the baseline design for the acceleration system assumes a high-gradient straight linac followed by several FFAG rings. In this paper the concept of a Neutrino Factory based on a highgradient proton Linac that is also used for acceleration of muons will be presented. We beleive that the incremental cost to upgrade the superconducting proton driver linac to be able to accelerate muons, including the costs to produce and cool the muons, will be considerably less than the costs estimated in previous neutrino factory design studies. We also believe that such an approach can produce a much higher neutrino flux and, because of the necessity for effective muon cooling, also be on the path to an energy frontier muon collider.

\section{Baseline Proton Linac Design}

The present design of a proposed superconducting 8 $\mathrm{GeV}$ Linac proton driver has a front end that uses a combination of $1300 \mathrm{MHz} \beta<1$ elliptical cavities and $325 \mathrm{MHz}$ spoke resonators. The high energy (from $\sim 1.2 \mathrm{GeV}$ and up, $\beta=1$ ) portion of the linac is made out of nine cell $1300 \mathrm{MHz}$ TESLA type cavities. Figure 1 shows a picture and diagram of a superconducting TESLA cavity.

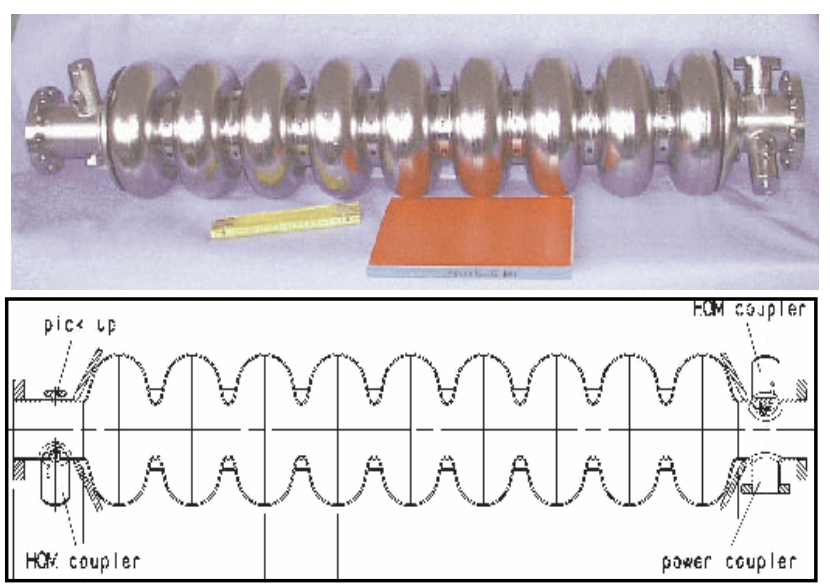

Figure 1. Picture and diagram of a TESLA RF cavity.

Each cryomodule contains a group of eight cavities and there is a quadrupole magnet between cryomodules. Each cavity is independently powered with independent phase and amplitude control using high power YIGferrite phase shifters. In its initial configuation, the high energy part of the linac (from $1.2 \mathrm{GeV}$ to $8 \mathrm{GeV}$ ) requires only 11 klystrons. The proton linac up to $100 \mathrm{MeV}$ is powered with $325 \mathrm{MHz}$ klystrons. The $\mathrm{H}^{-}$ beam peak current is $25 \mathrm{~mA}$ and each beam pulse is $1 \mathrm{~ms}$ long. Up to $2 \times 10^{14}$ protons are accelerated per pulse with a $5 \mathrm{~Hz}$ repetition rate as it is presently envisioned. 


\section{Repetition Rate Improvements}

One very important point of this paper is that the repetition rate of the Linac can be increased to great advantage for muon applications. The muon lifetime, even considering the Lorentz boost, is much shorter than the repetition period (e.g. a neutrino factory lifetime is about one $\mathrm{ms}$ and for a $2.5 \mathrm{TeV}$ on $2.5 \mathrm{TeV}$ muon collider the lifetime is $50 \mathrm{~ms}$ ). In the case of a collider, since the luminosity diminishes as the square of the intensity, a practical store would only last one lifetime and a repetition rate of $20 \mathrm{~Hz}$ would be ideal. In the case of a neutrino factory, the neutrino flux would be proportional to the repetition rate up to almost $1 \mathrm{kHz}$. Of course this potential factor of 200 in neutrino flux would be limited by the power capabilities of the production target (or targets if they are fed in parallel) of the muon cooling energy absorber, and the RF systems. Considering these cases and the other potential uses for protons at the laboratory, it will probably be most efficient to increase the cryomodule refrigeration to allow the RF systems to run continuously rather than be pulsed.

\section{Targeting, Cooling, and Preacceleration}

Using multi turn injection the $\mathrm{H}^{-}$beam is injected into an $8 \mathrm{GeV}$ storage ring (perhaps the Main Injector or Recycler Ring). The beam is then bunched at a subharmonic of $1300 \mathrm{MHz}$ and targeted to produce muons that are bunched and cooled [3]. If additional emittance reduction is required beyond what is possible in the cooling section, some preacceleration can be added using $200 \mathrm{MHz}$ and/or or $325 \mathrm{MHz}$ superconducting cavities. We also assume that the process of accumulating and preparing the proton beam for targeting will take more than 300 microseconds which is the time needed for the phase shifters in the Linac to adjust cavity phases for muon acceleration. The peak muon current would be $25 \mathrm{~mA}$, the same as for protons.

As a first step to study the use of the proton Linac to accelerate muons, the acceptance of the Linac for a large emittance muon beam has been simulated using the proton beam quadrupole settings. Figures 2 and 3 are Trace3D plots of the beam envelopes for protons and muons, where the optimum proton settings were used for both plots. The admittance at the injection point for muons is $22.5 \mu \mathrm{m}$. At $2 \mathrm{GeV}$ as assumed in figure 3, the muon beam normalized transverse emittances must therefore be cooled to less than $450 \mu \mathrm{m}$, which should be easily reached with the techniques now being simulated. The longitudinal matching of the cooled muon beam to the Linac acceptance should be straight forward as the HCC longitudinal cooling should be quite good.

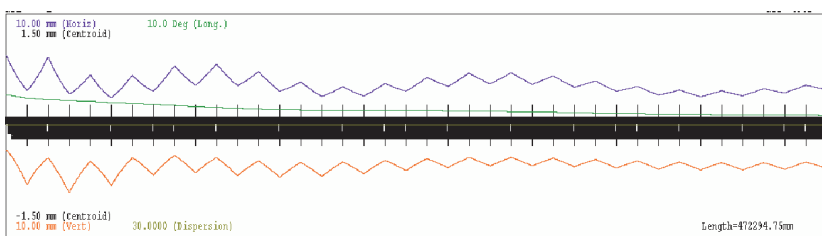

Figure 2. Trace3D display of the proton beam envelope for the 1.2 to $8 \mathrm{GeV}$ section of the proposed proton driver superconducting Linac. The blue is the horizontal and the red the vertical size as measured from the center of the plot, where the scale is $\pm 10 \mathrm{~mm}$.

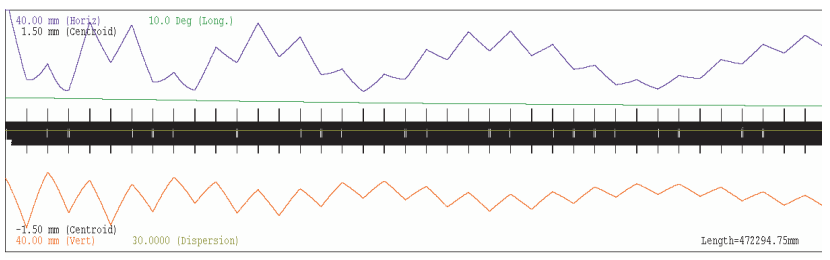

Figure 3. The same plot as in figure 2 for a muon beam that is injected at $2 \mathrm{GeV}$ with the same quadrupole settings as used for the proton beam. Note the scale is increased a factor of 4 to $\pm 40 \mathrm{~mm}$ compared to figure 2 .

\section{Discussion}

The original arguments for a superconducting proton driver Linac were to provide a versatile source of protons for a variety of future programs and to get Fermilab a practical start with the ILC technology. The option of also using it to accelerate muons after they have been produced by the same proton driver has been made much more plausible by recent innovations in muon cooling.

A neutrino factory based on a muon storage ring which could be filled at a much higher repetition rate than has previously been imagined would be a superior research instrument, surely better than anything now being discussed. The cost of increasing the repetition rate and to operate the Linac in CW mode and the improved neutrino flux will be compared to the previous neutrino factory cost studies and flux estimates. The development of muon acceleration capabilites as implied here will make an energy frontier muon collider a compelling future machine for high energy physics.

\section{References}

\footnotetext{
* Work supported in part by the U.S. Dept. of Energy through the Univ. Research Association under contract DE-AC35-89ER40486

[1] G. W. Foster and J. A. MacLachlan, Proceedings of LINAC 2002, Gyeongju, Korea

[2] R. P. Johnson, Technical Challenges of Muon Colliders, this workshop.

[3] K. Paul et al., Working Group 3, this workshop.
} 